\title{
Stealth Adaptation of Viruses: Implications for Therapy and for Potential Toxicity of Vaccines
}

\author{
W John Martin* \\ Institute of Progressive Medicine, USA \\ Submission: September 22, 2015; Published: September 28, 2015 \\ *Corresponding author: W John Martin, Institute of Progressive Medicine, 1634 Spruce Street, South Pasadena CA 91030, USA, Tel: 626-616- \\ 2868; Email: wjohnmartin@ccid.org
}

\begin{abstract}
The scope of human and animal virology research is likely to undergo a major expansion. It will occur with the realization that the immune system may fail to effectively recognize derivative viruses, which no longer express the major antigens normally targeted by cytotoxic $\mathrm{T}$ lymphocytes (CTL). This immune evasion mechanism has been termed stealth adaptation. Persistent infections with stealth adapted viruses can lead to various illnesses and especially those with neuropsychiatric symptoms. Certain stealth adapted viruses almost certainly entered into humans via monkey cytomegalovirus contaminated polio vaccines. Preexisting stealth adapted virus infections may render individuals susceptible to disease provocation from administered vaccines. On a more promising note, the research has helped identify an alternative cellular energy (ACE) pathway that can suppress both stealth adapted and conventional viruses. The ACE pathway is providing a new therapeutic paradigm that extends beyond virology to general medicine. It is also leading towards a basic understanding of how Nature prevents the fusion of opposite electrical charges. It is postulated that Nature does so through a fundamental force, tentatively termed KELEA (kinetic energy limiting electrostatic attraction).
\end{abstract}

Keywords: ACE, alternative cellular energy, African green monkey simian cytomegalovirus, AIDS, autism, brain, cytomegalovirus, inflammation KELEA, PCR, polymerase chain reaction, polio vaccine, SCMV, stealth adapted viruses

Abbreviations: ACE: Alternative Cellular Energy; CFS: Chronic Fatigue Syndrome; CPE: Cytopathic Effect; CSF: Cerebrospinal Fluid, CTL: Cytotoxic T Lymphocyte; ICE: Insufficiency Of Cellular Energy; HPV: Human Papillomavirus; KELEA: Kinetic Energy Limiting Electrostatic Attraction; PCR: Polymerase Chain Reaction; UV: Ultraviolet.

\section{Introduction}

Inflammation is generally regarded as an essential component of an effective defense mechanism against virus infections [1]. While it can be directly evoked by virus induced cellular damage, inflammation is mainly viewed as an outcome of cellular immunity [2] and, in particular, T lymphocyte recognition of virus coded components (antigens). Since each individual T lymphocyte is genetically programmed to recognize only a single antigenic specificity [3], effective lymphocyte engagement with a virus infected cell requires multiple copies of the recognized antigen to be displayed on the surface of the infected cells. There are additional requirements for a virus component to be recognized by T lymphocytes, including the need to bind to a particular region of the major histocompatibility complex (MHC) of the infected cell [4]. These restrictions account for the finding that relatively few virus components actually comprise major targets for the cellular immune response. Among the best examples is human cytomegalovirus (HCMV) in which one component (coded by the UL83 gene) is targeted by over $50 \%$ of anti-HCMV cytotoxic T lymphocytes (CTL) [5]. The majority of the remaining responding CTL are directed against either the UL55 or UL123 gene coded antigen [6]. Another example of immune evasion is with hepatitis $B$ virus (HBV), in which a mutation can occur in the gene coding the CTL targeted hepatitis surface antigen [7]. This example is not as clear as with HCMV, however, since the nucleotides coding the surface antigen also code the DNA polymerase gene, often rendering the mutant virus less virulent and less sensitive to anti-viral therapy [7]. A generic immune evasion mechanism that results from the loss or mutation of components normally targeted by CTL has been termed stealth adaptation [8-11]. A lesser form of this mechanism can be antigenic loss or mutation that primarily limits immunological recognition in those individuals with certain MHC specificities.

The existence of stealth adapted viruses has shed important new light onto many important aspects of human and animal health. This review i) provides a brief introduction to the early identification of stealth adapted viruses, including evidence that some are derived from African green monkey simian cyto 
megalovirus (SCMV); ii) summarizes data on polio vaccines as one of the sources of stealth adapted viruses now infecting humans and the possible cause of the emergence of the human immunodeficiency virus (HIV); iii) suggests that stealth adapted viruses are a potential cause of many human diseases, including autism, chronic fatigue syndrome (CFS), mental illnesses, Alzheimer's disease and certain cancers; iv) explains the potential risks that vaccination can pose for individuals with a preexisting stealth adapted virus infection; v) emphasizes that the body is still able to suppress both stealth adapted and conventional viruses through an energy based defense mechanism referred to as the alternative cellular energy (ACE) pathway. Some examples of ACE pathway based therapies for conventional viruses, including diseases caused by herpes simplex virus (HSV), herpes zoster virus (HZV), human papillomavirus (HPV) $[12,13]$, rotavirus [14] and HIV [15] are provided in the cited references.

\section{Stealth Adapted African Green Monkey Simian Cytomegalovirus (SCMV)}

Evidence for active virus infections in CFS patients was initially obtained using the polymerase chain reaction (PCR) as a diagnostic tool [16]. In very early (pre-1990) studies, low stringency PCR using primers designed to broadly detect herpes viruses yielded weak but still positive findings in approximately $30 \%$ of CFS patients. Reactivity was also seen in the cerebrospinal fluid (CSF) repeatedly obtained from an infant with choroid hemorrhages, hepatomegaly and thrombocytopenia. He was suspected of having a viral illness at birth but routine virus cultures were negative. A positive PCR was also obtained on a 1990 stereotactic brain biopsy of a school teacher with declining cognition and whose MRI showed bilateral periventricular lesions [17]. In spite of the positive PCR, the brain biopsy was totally devoid of any inflammation, although cellular damage was apparent histologically and confirmed by electron microscopy. Even though intact virus particles were not seen, there was extensive vacuolization, mitochondrial disruption and unusual intracellular inclusions, consistent with virus pathology [17]. Because of the lack of any discernable inflammation, the putative virus being detected by the PCR was designated as being a stealth virus. The same designation was used to explain positive PCR results on several acellular CSF samples obtained from patients with severe, chronic neurological illnesses and on blood samples from CFS patients.

Through serendipity, an additional primer set originally matched to a retrovirus was also tested in some of the patients. It yielded a strikingly strong PCR using blood samples from a 43-year-old woman recently hospitalized to rule out meningitis/ encephalitis. The woman subsequently became chronically disabled with a diagnosis of CFS. While prior viral cultures on CFS patients were providing suggestive results, a determined effort was made to culture the blood of the strongly positive PCR patient. After several weeks, fibroblasts inoculated with cells from her blood displayed an overt cytopathic effect (CPE); easily transmitted to secondary cultures (17). Electron microscopy confirmed abundant virus particles, along with extensive vacuoles and inclusions [18]. A similar very strongly positive culture was obtained from the CSF of a 21-year-old female patient admitted in coma to the Los Angeles County Hospital. The patient had a 4-year history of a severe manic-depressive (bipolar) psychosis [19]. Remarkably, her CSF was essentially acellular and her coma was clinically attributed to anoxia from a drug overdose. Aliquots of the positive culture were sent to the Los Angeles County Public Health Laboratory. They simply confirmed the CPE and lack of staining by available virus typing antisera. Minor changes in the culturing procedures led to more rapid and consistently positive cultures in CFS patients referred by Dr. Jay Goldstein, a physician specializing in CFS patients. The culture CPE was characterized by the formation of foamy, vacuolated cells with syncytia [18]. The finding of positive cultures in CFS patients was reported to the Los Angeles County Public Health Laboratory.

PCR generated products from the strongly positive culture of the 43-year-old CFS patient were cloned and sequenced. The sequencing identified two distinct nucleotide sequences, each flanked at both ends by the same primer [18]. The sequences of one of the clones showed highly significant matching, but not complete homology, to HCMV sequences available on GenBank [18]. At the time, GeneBank contained no significant matching sequences to the other clone. DNA extracted from pelleted culture supernatant, with and without subsequent agarose banding, was also cloned into plasmids. Sequencing allowed for the design of additional PCR primers that yielded strongly positive reactions when tested on repeated cultures from the patient and also on the CSF and on the CSF culture of the patient with the bipolar illness [19]. Other patients' strongly positive cultures tested negative using the same sets of primers, indicating that different viruses could undoubtedly undergo stealth adaptation.

Sequencing of additional clones from the culture DNA allowed for more comparisons with the available data on GenBank. Near perfect matching was obtained between some of the cloned DNA and the DNA of African green monkey simian cytomegalovirus (SCMV) [20]. Since African green monkeys were used in the manufacturing of polio vaccines, the results were reported to the Food and Drug administration (FDA), Centers for Disease Control and Prevention (CDC) and the polio vaccine manufacturer.

Finer sequencing of the DNA clones showed considerable genetic instability, with mutations, deletions and recombinations [21]. Evidence for the genes corresponding to UL55 and UL83 in HCMV were not found, although the cloning may have failed to capture the UL83 gene because of a paucity of restriction sites of the enzymes used in the cloning. Major mutations were noted in the sequenced region to the SCMV gene corresponding to UL123 in HCMV [9]. The virus genome was also apparently fragmented, with aggregate sequences far beyond the approximate 20 kilobase size of the extracted DNA on agarose [18]. Other data raised the possibility of DNA fragments being bridged by RNA sequences. Moreover, additional sequences were identified in the cloned DNA of purified virus culture supernatant. Some of these sequences matched to cellular DNA [22], while others matched to bacteria [23]. The latter observation is particularly important since it may explain some of the atypical bacteria isolated from culture positive patients [24].

The cultured virus is pathogenic when inoculated into cats producing an acute, severe generalized disease, with marked neurobehavioral disturbance [25]. Yet, there was no 
inflammation, justifying the notion of the virus being stealth. Yet from about 6 weeks to 16 weeks when the last cat was necropsied, the remaining cats showed a rather remarkable clinical recovery.

\section{Cytomegalovirus Contamination of Polio Vaccines}

The prospect of developing a polio vaccine was given an enormous boost when John Enders and co-workers showed that the polio virus could be successfully cultured [26]. Their tissue culture efforts were facilitated by the earlier discovery of antibiotics. An initial concern in the development of polio vaccines was that any established cell line might be perceived as having undergone genetic changes towards becoming cancerous. In spite of publicly aired advice to the contrary, the decision was, therefore, made to use primary cultures of monkey kidney cells. Rhesus monkeys were initially used by the three early pioneers, Drs. Jonas Salk, Albert Sabin and Hilary Koprowski [27]. Dr. Salk opted to use formalin inactivated viruses as had been used in influenza vaccine development. Because the virus was to be inactivated, he chose to use the virulent disease causing virus strains. The other two researchers opted to use attenuated (less virulent) strains, as modeled after the yellow fever vaccine. Dr. Sabin followed the suggestion of Dr. Roberto Dulbecco and plaque-purified his isolates, gaining an advantage over the work of Dr. Koprowski. Dr. Salk was given the upper hand in the United States, while Dr. Sabin focused his work in the Soviet Union. Dr. Koprowski did studies in both Europe and Africa [27].

The United States preference for Salk vaccine was consistent with concerns expressed by several prominent virologists that using cells directly cultured from monkeys to produce noninactivated polio viruses posed risks for contamination with monkey viruses. In their competition, Dr. Sabin both published and personally communicated with Dr. Kopowski that the CHAT vaccine produced in rhesus monkeys was contaminated with a difficult to culture cytopathic virus [28]. The CHAT vaccine was subsequently shown to have DNA of rhesus cytomegalovirus (RhCMV) [29]. The CHAT vaccine was extensively tested in chimpanzees, many of which became sick, as did some of the African animal handlers. A plausible case has been made that HIV arose from some of the CHAT vaccine inoculated chimpanzees [29].

A setback to Dr. Salk's vaccine was that the virus inactivation process was not always successful and polio outbreaks began to occur during springtime, which some attributed to the experimental use of the vaccine. This issue became more transparent with the licensure of the Salk vaccine when many children became infected with an inadequately inactivated vaccine. A bigger setback was the reluctant acceptance that even with standard inactivation, the Salk vaccine still contained a polyoma-like virus. It was identified as simian virus-40 (SV-40), prevalent in rhesus monkeys [27].

Two major changes then ensued in the early 1960's. The first was to switch from using rhesus monkeys to the use of African green monkeys. The second was to adopt Dr. Sabin's live, attenuated polio strains [27]. The US Government was challenged by a pharmaceutical company that if they were to license Dr. Sabin's vaccine, they would have to license Dr. Koprowski's vaccine. Even though it was being used overseas, the US Government was aware of the questionable virulence and additional contamination issues of Dr. Kopowski's vaccines. The Government appealed to Dr. Sabin and he agreed that he would freely provide his vaccine strains to Lederle as a means of avoiding the forced US licensure of Dr. Kopowski's vaccine.

The rather abrupt switch from rhesus to African green monkeys and from inactivated to live virus was a continuing cause of concern, even to workers within FDA's Bureau of Biologics. An employee's suggestion that sera from the monkeys be tested for antibody reactivity on the cultured cells as a marker for likely virus contamination was opposed by those in authority. Still in 1972, an earnest effort was made to more closely examine monkey kidney cell cultures. Rather than being used for vaccine production, kidney cell cultures from eleven monkeys were examined for contaminating viruses. All eleven cultures produced SCMV, with only 4 of the positive cultures being detectable using the routine screening assay. The vaccine manufacturer formulated contingency plans to respond to any actions of the FDA to the findings of their joint study. A major argument was that extensive experience with the vaccine had not shown signs of any untoward acute infections. Another argument was that contaminating SCMV would be destroyed during passage through the stomach. Possibly the most telling comment was the expressed opinion that FDA would not have the courage to take their product off the market.

Disregarding the emerging evidence for delayed acting (slow) virus-like illnesses, FDA resolved the issue by suggesting if the vaccine cultures were harvested quickly enough and cultures inoculated with anti-polio neutralized vaccine were examined early enough, evidence for SCMV could be avoided. With the later publication of SCMV DNA still being present in vaccines produced after 1972 [29,31], this approach was clearly ineffective.

One of the FDA officials involved in the earlier study was informed in 1978 of foreign (non-monkey) DNA being present in polio vaccine lot. The information was summarily dismissed by the FDA official with the comment "every time you eat an apple you consume foreign DNA". The particular vaccine lot (3-444) was selected because polio virus neutralized vaccine induced an atypical CPE and showed evidence of reverse transcriptase, initially suggestive of a possible retrovirus. Further studies helped dismiss the likelihood of a retrovirus and the vaccine lot was approved.

Reports of stealth adapted SCMV contributed to efforts to switch back to using formalin inactivated vaccines. There was also the more politically accepted argument that the live, attenuated vaccine can very occasionally revert to a more virulent strain [32]. Still, the problem is that once a virus is introduced into humans, it can persist due to human to human transmission, and in the case of stealth adapted viruses, reciprocal human to animal transmissions. A very major concerned with down-sized stealth adapted viruses is the possibility of virus carriage through bacteria. 


\section{Juniper Online Journal of Immuno Virology}

\section{Illnesses Attributed to Stealth Adapted Viruses}

Among the body's organs, the brain is unique in the spatial non-uniformity of its many functions and the cooperative networking between different regions. Even limited localized damage to the brain can, therefore, be far more functionally disruptive than direct virus damage to other organs. While stealth adapted virus infection is widespread in the body, as in the inoculated cats, it is not surprising, therefore, that the predominant manifestations are neurobehavioral. During several years of culturing for stealth adapted viruses, it was truly exceptional to obtain negative cultures in patients with severe neurological and/or psychiatric illnesses. While typically not as striking and certainly not as frequent, positive results were occasionally found in apparently symptom-free individuals. In several blinded studies, about $10 \%$ of control cultures were identified as being clearly positive. A similar incidence of positive cultures was obtained in a study performed in 2002 on blood donors at the University of California Irvine. The implication of this finding in terms of safety of the Nation's blood supply led the CDC to sponsor efforts to limit further clinical testing.

Many viruses can cause illnesses in only a subset of infected patients. With polio virus, it is generally estimated that only one percent of those infected develop neurological symptoms [27]. If a cytopathic virus is isolated from the blood and particularly from the CSF of a patient with a neuropsychiatric illness, the virus should be considered a likely contributing factor to the patient's illness until proven otherwise.

A useful insight emerged from the virus studies. It was that PCR and immune typing assays on different cultures yield variable results consistent with stealth adaptation being a generic process, potentially occurring with all human and animal viruses. The heterogeneity of the origins of stealth adapted viruses, along with evidence of genetic deletions and mutations, can render PCR and immune typing assays somewhat unreliable when performed on patients' blood samples. A negative PCR assay directed to a limited region within a virus type, does not necessarily exclude the possibility that other regions of that type of virus are present or that another type of virus is infecting the patient. Some stealth adapted DNA viruses may revert to replicating RNA forms such that a reverse transcription step needs to be included in the PCR assay [33]. Genetic instability of stealth adapted viruses [21] can also yield antigens with a broader range of immune reactivity than seen with the particular type of virus from which the stealth adapted virus is derived. Mycoplasma and alphaproteobacteria sequences were identified in cultures of the prototype SCMVderived stealth adapted virus [10]. The likelihood of bacterial sequences in other stealth adapted viruses may explain misleading positive assays for Borrelia burgdorferi, (the cause of Lyme disease) and/or for Mycoplasma fermentans, as variously reported in CFS patients $[34,35]$. For these reasons, the most reliable detection method for stealth adapted viruses still remains the culturing of blood, CSF and tissue samples from patients and observing for a characteristic CPE, which tends to repair with the production of extracellular materials. The prototype stealth adapted virus grows readily in cell lines from multiple species and has even been grown on an insect cell line. This is consistent with interspecies transfer of infection between patients and their domestic pets [25].

While the majority of patients on whom blood testing was performed were diagnosed as having CFS, autism or a major neuropsychiatric illness [17, 36-38], additional studies involved other patient groups. For example, positive cultures were regularly seen in patients with multiple myeloma [38]. Upon review, many multiple myeloma patients have prior or even ongoing neurocognitive impairments, not typically addressed by their hematologist [39]. Hodgkin's disease, aggressive lymphomas, glioblastoma, breast and salivary gland tumor patients [40] have also provided examples of strikingly positive stealth adapted virus cultures. Again upon clinical review, mental fatigue and other indicators of brain dysfunction, not uncommonly preceded the patient's cancer diagnosis. Post chemotherapy fatigue is explainable as an adverse effect of therapy, but breast cancer patients commonly report persisting fatigue even after the cancer has been excised and no chemotherapy administered (personal communication from a patient support group).

A social contact of the female patient from whom the prototype SCMV-stealth adapted virus was isolated, was experiencing many of the same neurocognitive difficulties as the patient. Their viral cultures were essentially identical in appearance. While the female patient was HIV negative, her friend was HIV positive and died shortly thereafter. Positive stealth adapted virus cultures have also been seen in other HIV infected patients and may contribute to the pathogenicity of HIV [41] and particularly to the neuro cognitive impairments seen in many AIDS patients [42].

Evidence of non-sexual transmission of stealth adapted viruses has also been observed in community, workplace, family and personal interactions. A significant epidemic, reported to Public Health authorities, occurred in 1996 in Needles, California and in the adjacent Mohave Valley [43]. The epidemic may have originated from returning veterans since several were included among the earlier identified patient population. Included in the epidemic was an infected child who was initially diagnosed as having a behavioral disorder. A detailed neurological examination was conducted after he informed his physician mother of temporarily seeing double (diplopia) [44]. A biopsy of his brain was obtained on the basis of periventricular lesions being seen on MRI. The biopsy showed several foamy vacuolated cells, but without accompanying inflammation [44]. A second brain biopsy was also examined by electron microscopy. It confirmed marked mitochondrial disruption and structured intracellular inclusions, again in the absence of any inflammation [45]. His virus cultures were repeatedly positive, as were his mother's. Although he responded initially to ganciclovir, he subsequently died within a year of having the first brain biopsy. The mother, a Gulf War veteran, has been persistently ill from the time of the epidemic.

Another virus culture patient believed he acquired an illness from non-intimate socializing during a business trip. He was initially concerned that he had contracted HIV but tested negative. He became more concerned when he realized his son was not behaving normally and that after a weekend visit, his 
father became ill. He surreptitiously had his blood and that of his father tested for SCMV by PCR at a primate virology laboratory. Both samples were reported as being positive for SCMV. He also tested positive in culturing for stealth adapted viruses. An employee at the CDC was informed of the results and arranged for a blood sample to be sent to an outside laboratory, which presumably failed to obtain positive results.

Based on positive virus cultures of many children with autism [46] and on some of the mothers, it is confidently postulated that transplacental and possibly postnatal transmission of stealth adapted viruses can lead to the impaired limitations in social interactions, including language skills that characterizes autism. Stealth adapted virus infection can also explain the relatively high frequency of epilepsy seen in children with autism. The severity and actual manifestations of disease are likely influenced by the areas of the brain that are infected and also by the innate and learned strengths of the child in engaging in social interactions. Less severe congenitally or later acquired childhood illness can explain the increasing prevalence of learning and behavioral problems in school age children and some criminal behaviors in adults.

\section{Provocation Encephalitis}

Reports of illness occurring shortly after receiving vaccines are officially dismissed on the basis of statistical data that such events may be coincidental. Rarely is serious consideration given to the prospect of vaccine provocation of an underlying stealth adapted virus infection [47]. Purified microbial vaccine antigens are not inherently highly immunogenic and immuneboosting adjuvants are commonly co-administered with the vaccine. The concurrent use of multiple vaccines, each with its own adjuvant, can potentially result in unnecessarily excessive immune stimulation. Stealth adapted viruses may well continue to express components, which in the strong adjuvant setting of vaccination, may become recognizable as antigens by the strengthened cellular immune system. Vaccination could, thereby, provoke an illness, which would likely have neuropsychiatric manifestations. Another scenario can occur with administering live virus vaccines. It is that co-infecting viruses may potentiate the growth and pathogenicity of stealth adapted viruses. It would be worthwhile to analyze the medical history of vaccine damaged children, including recipients of HPV vaccines for evidence of prior illness consistent with a preexisting stealth adapted virus infection. The Public Health system should review the likely excessive use of adjuvants when multiple vaccines are simultaneously administered. It should also examine the benefits of each of the many recommended vaccines in patients, who by history or laboratory testing, show evidence for being stealth adapted virus infected.

\section{Alternative Cellular Energy (ACE) Pathway}

Probably the most important insight gained from studies on stealth adapted viruses is that the body is not totally dependent upon the immune system to suppress virus infections. Nonimmunological suppression of stealth adapted viruses was first noted in virus cultures [48]. The CPE in positive cultures regularly undergoes a repair process, which is reversed by replacing the tissue culture fluid (medium) with fresh medium. The repair is attributed to the accumulation of cell derived materials, which tend to self-assemble into particles, ribbons and threads. Atypical intra- and extracullular structures can also be seen in brain cells from infected humans $[17,45]$ and inoculated cats [25]. Similar materials are also present on skin and attached to hair of certain patients, presumably forming by the aggregation of materials released in perspiration [49]. The culture-derived and patient derived pigmented materials display various energyrelated properties. In particular, the particles are; i) electrostatic, consistent with their tendency to self-assemble; ii) fluorescent under ultraviolet (UV) light, especially when interacting with certain dyes, including neutral red and acridine orange; iii) occasionally ferromagnetic and iv) most strikingly capable of preventing reactivation of stealth adapted virus induced CPE when added to the re-feeding medium [48]. A particularly striking observation when examining brain biopsies of infected patients is the very marked disruption of the cells' mitochondria $[16,45]$. Given this, a reasonable assumption is that the materials are allowing cell survival by bypassing the dependency on mitochondrial metabolism. The materials were, accordingly, termed alternative cellular energy (ACE) pigments [48]. An ACE pathway was envisioned as being a source of energy distinct from mitochondria oxidative phosphorylation of food nutrients. Since a few ACE pigment particles could affect the re-feeding medium, it was considered likely that they were mediating their effects by modifying a property of the culture medium.

Gas chromatograph - mass spectroscopy (GC-MS) analyses of ACE pigments showed a diverse range of relatively simplealiphatic and aromatic chemicals. Energy-dispersive x-ray spectroscopy (EDX) indicated that individual particles contain relatively few minerals, but in aggregate many minerals were identified [48]. The cultures were also characterized by lipid production in the form of long needles, linear troughs, crystal, and pyramid shaped structures [42]. The lipid production continued long after all of the cells had died. Similar lipid production has been seen with particles derived from dried perspiration of stealth adapted virus infected patients [48]. The abiotic synthesis of lipids is extremely interesting with regards to the origin of life.

Patient derived ACE particles in water can lead to the slow formation of gas bubbles [49]. Patient and culture derived ACE particles have also been shown to reduce the surface tension and specific heat of water and to increase its volatility (unpublished). These actions have now been explained by the capacity of ACE particles to attract an environmental force termed KELEA (kinetic energy limiting electrostatic attraction) and transfer the energy to water, resulting in a loosening of the intermolecular hydrogen bonding [50]. Once water is sufficiently activated, its separated charges can then directly absorb KELEA from the environment, leading to further activation of the water [51]. As noted above, the ACE pathway is viewed as a naturally occurring mechanism that enables cells to derive energy apart from that provided through food metabolism. The production of readily identifiable ACE pigments is likely the response to an insufficiency of cellular energy (ICE); a cause of which can be the increased energy demands caused by an infection. Evoked 


\section{Juniper Online Journal of Immuno Virology}

fluorescence within the skin of an elderly patient being treated by neutral red dye for shingles suggested that ACE pigments may be distributed via nerves to localized areas of ICE (unpublished). Transport may also occur within circulating blood cells. The basic notion, however, is that all cells have the potential capacity to utilize the ACE pathway through the production of ACE pigments. The resulting enhanced kinetic activity of the intracellular fluid is thought to facilitate normal metabolic activities and, thereby, help reduce the severity of disease. Conversely, factors that can inhibit the functioning of the ACE pathway may underlie disease exacerbations. An exciting possibility is that electrical activity of the brain may essentially act as a variable antenna, able to absorb KELEA into the body [52]. Indeed, a direct relationship may exist between the brain and activation of the body's fluids. Consuming KELEA activated ACE Water may provide an expedient way to help ensure a positive feedback relationship. These concepts are helping to open a new paradigm of testable therapies, as will be discussed in future articles.

\section{Summary}

This review is intended to help lay the foundation for those unfamiliar with the concept of stealth adaptation of viruses. While the existence of stealth adapted viruses poses both political and scientific challenges to Public Health authorities, there is a major role for virologists to help in further characterizing their origins, host range, replication, transmission and pathogenicity. While showing a limitation of the immune system in virus defense, the study of stealth adapted viruses has revealed a novel alternative cellular energy (ACE) pathway that can be utilized in suppressing both stealth adapted viruses and the conventional viruses from which they are derived.

\section{Acknowledgement}

The Institute of Progressive Medicine is a component of Ml Hope Inc., a non-profit public charity.

\section{References}

1. Ward PA(2010) Acute and Chronic Inflammation in Fundamentals of Inflammation Ed. Charles N. Serhan, Peter A. Ward, Derek W. Gilroy Cambridge University Press pp 1-16.

2. Neal Nathanson 2007 Viral Pathogenesis and Immunity Academic Press pp 280.

3. Burnet FM (1959) The Clonal Selection Theory of Immunology Nashville Vanderbilt University Press.

4. Doherty PC, Zinkernagel RM (1975) H-2 compatibility is required for T-cell-mediated lysis of target cells infected with lymphocytic choriomeningitis virus. J Exp Med 141(2): 502-507.

5. Wills MR, Carmichael AJ, Mynard K, Jin X, Weekes MP et al. (1996) The human cytotoxic T-lymphocyte (CTL) response to cytomegalovirus is dominated by structural protein pp65: frequency, specificity, and T-cell receptor usage of pp65-specific CTL. J Virol 70: 7569-7579.

6. Borysiewicz LK, Hickling JK, Graham S, Sinclair J, Crange MP, et al. (1988) Human cytomegalovirus-specific cytotoxic T-cells, Relative frequency of stage-specific CTL recognizing the $72 \mathrm{kD}$ immediate early protein and glycoprotein B expressed by recombinant vaccinia viruses. J Exp Med 168(3): 919-931.

7. Tong S, Li J, Wands JR, Wen YM (2013) Hepatitis B virus genetic variants: biological properties and clinical implications. Emerg Microbes Infect 2(3): e10.

8. Martin WJ (1994) Stealth viruses as neuropathogens. CAP Today 8(10): 67-70.

9. Martin WJ (1999) Stealth adaptation of an African green monkey simian cytomegalovirus. Exp Mol Pathol 66(1): 3-7.

10. Martin WJ (2014) Stealth adaptation of viruses: Review and updated molecular analysis on a Stealth adapted African green monkey simian cytomegalovirus (SCMV). J Hum Virol Retrovirol 1(4): 00020.

11. Martin WJ (2014) Stealth Adapted Viruses; Alternative Cellular Energy (ACE) \& KELEA Activated Water. Author House Bloomington, IN pp 321. ISBN 971-1-4969-0496-6.

12. Martin WJ, Stoneburner J (2005) Symptomatic relief of herpetic skin lesions utilizing an energy based approach to healing. Exp Mol Path 78(2): 131-134.

13. Martin WJ, Stoneburner J (2014) Alternative Cellular energy (ACE) pathway activation as the Mode of action of neutral red dye phototherapy of human viruses. J Hum Virol Retrovirol 1(4): 00019.

14. Izaguire RR, Guzman MR, Fuentes RC, Mena CE, Penate E, et al. (2014) Alternative cellular energy based therapy of childhood diarrhea. In: Martin W.J, Stealth Adapted Viruses; Alternative Cellular Energy (ACE) \& KELEA Activated Water. Author House, Bloomington IN. pp 103-114.

15. Dubrov V, Dubrova T, Christner D, Laurent D, Martin WJ (2015) Alternative cellular energy based therapy using enercel in advanced AIDS patients co-infected with tuberculosis and treated in Chernigov, Ukraine. J Hum Virol Retrovirol 2(6): 00061.

16. Martin WJ (1992) Detection of viral related sequences in CFS patients using the polymerase chain reaction. in "The Clinical and Scientific Basis of Myalgic Encephalomyelitis Chronic Fatigue Syndrome." Byron M. Hyde Editor. Nightingale Research Foundation Press. Ottawa Canada pp 278-283. ISBN 0-969-56620-4.

17. Martin WJ (1996) Severe stealth virus encephalopathy following chronic fatigue syndrome like illness: Clinical and histopathological features. Pathobiology 64(1): 1-8.

18. Martin WJ, Zeng LC, Ahmed K, Roy M (1994) Cytomegalovirus related sequences in an atypical cytopathic virus repeatedly isolated from a patient with the chronic fatigue syndrome. Am J Path 145: 440-451.

19. Martin WJ (1996) Simian cytomegalovirus related stealth virus isolated from the cerebrospinal fluid of a patient with bipolar psychosis and acute encephalopathy. Pathobiology 64(2): 64-66.

20. Martin WJ, Ahmed KN, Zeng LC, Olsen J C, Seward JG, et al. (1995) African green monkey origin of the atypical cytopathic 'stealth virus' isolated from a patient with chronic fatigue syndrome. Clin Diag Virol 4(1): 93-103.

21. Martin WJ (1996) Genetic instability and fragmentation of a stealth viral genome. Pathobiology 64(1): 9-17.

22. Martin WJ (1998) Cellular sequences in stealth viruses. Patobiology 66(2): 53-58.

23. Martin WJ (1999) Bacteria related sequences in a simian cytomegalovirus-derived stealth virus culture. ExpMol Path 66(1): 8-14.

24. Martin WJ (2005) Alternative cellular energy pigments from bacteria of stealth virus infected individuals. Exp Mol Path 78(3): 217-217.

25. Martin WJ, Glass RT (1995) Acute encephalopathy induced in cats with a stealth virus isolated from a patient with chronic fatigue syndrome. 


\section{Juniper Online Journal of Immuno Virology}

Pathobiology 63(3): 115-118.

26. Enders JF, Weller TH, Robbins FC (1949) Cultivation of the Lansing strain of poliomyelitis virus in cultures of various human embryonic tissues. Science 109(2822): 85-87.

27. Paul JR (1971) A History of Poliomyelitis. Yale University Press. pp 486.0-300-01324-8, ISBN 0-300-01324-8.

28. Sabin AB (1959) Present position on immunization against poliomyelitis. Br Med J 1 (5123): 663-680.

29. Baylis SA, Shah N, Jenkins A, Barry NJ, Minor PD (2002) Simian cytomegalovirus and contamination of oral poliomyelitis vaccines. Biologicals 31(1): 63-73.

30. Martin WJ (2015) Chimpanzees inoculated with cytomegalovirus contaminated polio vaccines may explain origin of HIV-1. J Hum Virol Retrovirol 2(2): 00035.

31. Sierra-Honigmann AM, Krause PR (2002) Live oral poliovirus vaccines and simian cytomegalovirus. Biologicals 30(3): 167-174.

32. Pliaka V, Kyriakopoulou Z, Markoulatos P (2012) Risks associated with the use of live-attenuated vaccine poliovirus strains and the strategies for control and eradication of paralytic poliomyelitis. Expert Rev Vaccines 11(5): 609-628.

33. Martin WJ (1997) Detection of RNA sequences in cultures of a stealth virus isolated from the cerebrospinal fluid of a health care worker with chronic fatigue syndrome. Case report. Pathobiology 65(1): 5760 .

34. Patrick DM, Miller RR, Gardy JL, Parker SM, Morshed MG, et al. (2015) Lyme disease diagnosed by alternative methods: A phenotype similar to that of chronic fatigue syndrome. Clin Infect Dis 61(7): 1084-1091.

35. Nicolson GL, Gan R, Haier J (2003) Multiple co-infections (mycoplasma, chlamydia, human herpes virus-6) in blood of chronic fatigue syndrome patients: association with signs and symptoms. APMIS 111(5): 557-566.

36. Martin WJ (1996) Stealth viral encephalopathy: Report of a fatal case complicated by cerebral vasculitis. Pathobiology 64: 59-63.

37. Martin WJ (2015) Stealth adapted viruses - Possible drivers of major neuropsychiatric illnesses including Alzheimer's disease. J Neurol Stroke 2(3): 00057.
38. Martin WJ (2015) Stealth adapted viruses. A bridge between molecular virology and clinical psychiatry. Open J Psychiatry 5(4): 311-317.

39. Durie BG, Collins RA, Martin WJ (2000) Positive stealth virus cultures in myeloma patients: A possible explanation for neuropsychiatric comorbidity. Blood (suppl 1) (2000) abstr 1553

40. Gollard RP, Mayr A, Rice DA, Martin WJ (1996) Herpes virus related sequences in salivary gland tumors. J Exp Clin Can Res 15: 14.

41. Martin WJ (2000) Chemokine receptor-related sequences in an African green monkey simian cytomegalovirus (SCMV)-derived stealth virus. Exp Mol Path 69: 10-16.

42. Brew BJ, Chan P (2014) Update on HIV dementia and HIV-associated neurocognitive disorders. Curr Neurol Neurosci Rep 14(8): 468.

43. Martin WJ, Anderson D (1997) Stealth virus epidemic in the Mohave Valley. Initial report of viral isolation. Pathobiology 65(1): 51-56.

44. Martin WJ, Anderson D (1999) Stealth virus epidemic in the Mohave Valley: Severe vacuolating encephalopathy in a child presenting with a behavioral disorder. Exp Mol Pathol 66(1): 19-30.

45. Martin WJ (2003) Complex intracellular inclusions in the brain of a child with a stealth virus encephalopathy. Exp Mol Pathol 74(3): 197 209.

46. Martin WJ (1995) Stealth virus isolated from an autistic child. J Autism Dev Disord 25(2): 223-224.

47. Martin WJ (2004) The rationale for vaccines and the potential inadvertent consequences including autism, AIDS and other epidemics. Med Veritas 1: 81-85.

48. Martin WJ (2003) Stealth virus culture pigments: A potential source of cellular energy. Exp Mol Path 74(3): 210-223.

49. Martin WJ (2005) Alternative cellular energy pigments mistaken for parasitic skin infestations. Exp Mol Path 78(3): 212-214.

50. Martin WJ (2015) KELEA: A natural energy that seemingly reduces intermolecular hydrogen bonding in water and other liquids. Open J Biophysics 5(3): 69-79.

51. Martin WJ (2015) Therapeutic potential of KELEA activated water. Int J Complement Alt Med 1(1): 00001

52. Martin WJ (2015) Is the brain an activator of the alternative cellular energy (ACE) pathway? Int J Complement Alt Med 1(1): 00002. 